\title{
Pruritus in Elderly Patients-Eruptions of Senescence
}

\author{
Timothy G. Berger, MD," and Martin Steinhoff, MD, PhD*,+
}

\begin{abstract}
Geriatric patients are frequently afflicted by pruritic dermatoses. Most pruritic elderly patients present with a skin eruption. The high prevalence of pruritic inflammatory skin disorders in elderly patients is a consequence of three physiological changes that occur with aging: (1) the epidermal barrier repair is diminished; (2) the immune systems of elderly patients are activated and have defective Th1 function along with enhanced Th2 function (immunosenescense); and (3) neurodegenerative disorders may lead to pruritus by their central or peripheral effects. These consequences of aging may all afflict the same patient, explaining why elderly people often have multiple overlapping skin conditions. The following article outlines the pathogenesis of the most common forms of pruritic skin disease in elderly patients and the hallmarks that allow the dermatologist to establish an accurate diagnosis and also suggests a management strategy for each common type of pruritic skin disease in the elderly patient.

Semin Cutan Med Surg 30:113-117 ( 2011 Published by Elsevier Inc.
\end{abstract}

$\mathrm{P}$ ruritus affects persons of all races and ages. However, in younger persons, usually the patient has either atopic dermatitis or urticaria or a self-limited but clearly defined skin disorder. In elderly patients (those older than 60 years of age and especially those in their 70s and 80s) pruritic eruptions are common. Unfortunately, they are often chronic and difficult to accurately diagnose. ${ }^{1}$ Recent scientific discoveries regarding physiological changes that occur in the aging process have shed some insight into why pruritic dermatoses would occur more commonly in elderly people, suggested diagnostic approaches, and helped identify effective therapies.

\section{The Physiology of Skin Aging}

Aging affects three important components involved in generating skin inflammation and in sensing pruritus: (1) the im-

* Department of Dermatology, University of California San Francisco, San Francisco, CA.

$\dagger$ Department of Surgery, University of California San Francisco, San Francisco, CA.

Dr. Berger has nothing to disclose and no conflict of interests to report.

Dr. Steinhoff has performed consultancy services for which he received compensation for Galderma, Merck, Sanofi-Aventis and Regeneron. On behalf of Dr. Steinhoff, his institution has received financial gifts from Maruho (Japan), and research was supported by ZymoGenetics, L'Oreal and Galderma.

Address reprint requests to Timothy G. Berger, MD, Department of Dermatology, University of California San Francisco, 1701 Divisadero Street, Room 341, San Francisco, CA 94143. E-mail: bergert@derm.ucsf.edu mune system; (2) the barrier function of the epidermis; and (3) the nervous system.

\section{Aging of the Immune System}

The changes that occur in the immune system with age are termed "immunosenescense." $2-5$ There are two general features of the aged immune system: (1) it is proinflammatory; and (2) there is significant aberration of the function of $\mathrm{T}$ and B cells. In some patients this results in an "allergic" phenotype, or an apparent Th2 dominance. ${ }^{6}$ The apparent cause of the proinflammatory state and the Th2 dominance is the loss of naive T cells, which reduce the T-cell repertoire. The immune effort required to control chronic viral infections, especially cytomegalovirus (in many people), and HIV (in those infected) results in loss of naive $T$ cells as the immune repertoire becomes populated with "committed" $\mathrm{T}$ and B cells. The loss of naive $T$ cells also reduces the ability of the aged patient to react effectively to infectious agents to which he/she has not been previously exposed. In addition, Th17 activity is well maintained in aging. ${ }^{7}$

\section{Aging and the Epidermal Barrier}

With age, there is significant change in the epidermal barrier. Starting at approximately age 55, the surface $\mathrm{pH}$ of the epidermis becomes less acidic. ${ }^{8}$ The enzymes required to process the lipids that compose the epidermal water barrier require an acid $\mathrm{pH}$. This is initially manifested as a reduced rate of barrier repair. Elderly patients frequently note irritation 
and pruritus from washing products and strategies that were well tolerated at a younger age. At around age 70, the rate of production of the precursors of the lipid barrier is reduced, resulting in insufficient lipids to maintain the barrier. Acid and neutral symphomyelinase, ceramide synthase, and acid ceramidase, enzymes required to produce ceramides with structural function in the epidermal barrier, are reduced in the inner layers of the epidermis in elderly people. ${ }^{9}$ Aquaporin-3 is a glycerol and water membrane channel that is critical in maintaining skin hydration by permitting adequate stratum corneum glycerol concentration. Aquaporin expression is linked to fillagrin degradation. Aquaporin-3 gene expression is reduced in persons older than 60 years of age. ${ }^{10}$ With all these defects in steps critical to epidermal barrier function and hydration, xerosis is consequently a major problem in elderly people. ${ }^{11}$ An impaired barrier has two other consequences: (1) Barrier failure or insufficiency may be related to increased risk for the development of contact dermatitis, because the impaired barrier may not prevent penetration of potential antigens into the epidermis; and (2) when the barrier fails, the cytokines released to induce barrier repair are proinflammatory, resulting in dermatitis. The relationship between fillagrin mutation and atopic dermatitis demonstrates this relationship between defective barrier and inflammatory skin disease.

\section{Degenerative Skeletal and Neural Disease}

Elderly patients frequently are afflicted with degenerative diseases of the spine. The degenerative disease can result in impingements on sensory nerves as they exit the spinal cord. Brachioradial pruritus and nostalgia paresthetica are conditions with this apparent pathogenesis. ${ }^{12}$ In addition, in rare cases, central nervous system neurodegenerative disease may produce itching. ${ }^{13}$ Diabetic truncal pruritus may be a symptom of diabetic polyneuropathy. ${ }^{14}$

\section{Practical Clinical Approach to the Elderly Patient with Pruritus}

The first step in evaluating an elderly patient with pruritus is to assess whether the patient has any primary skin lesions. If the patient has pruritus but no primary skin lesions, a separate investigative approach is pursued. Most elderly patients with pruritus have a visible inflammatory skin condition.

\section{Pruritus in the}

\section{Absence of Primary Skin Lesions}

This group of patients has one of two sets of conditions: an "invisible" dermatosis, or a systemic cause of their pruritus. ${ }^{15,16}$

\section{The "Invisible" Dermatoses}

These conditions have very subtle or only inducible skin lesions that are not obvious on a cursory examination. Xerosis is by far the most common of these conditions. The pathogenesis has been outlined previously in this article. The second consideration in elderly people is medication-induced pruritus (without a visible drug eruption). More than 100 medications have been reported to cause pruritus, probably by different mechanisms. For example, some medications induce hepatobiliary disease, and others (such as the angiotensin converting enzyme inhibitors) may induce urticaria. Opiates are increasingly used in elderly people to manage pain and may result in pruritus through direct interactions with the mast cells and itch-sensing neurons. A review of the patient's medications, looking for a temporal correlation between the onset of pruritus and starting of a new medication, is critical; however, the medication may have been started well before the itching began and itching may persist for months after the implicated medication is discontinued. Hydroxyethyl starch used in cardiac surgery can precipitate severe pruritus of prolonged duration with no visible skin lesions. Cutaneous T-cell lymphoma of the mycosis fungoides type, dermatitis herpetiformis, urticaria, lichen planus, fiberglass dermatitis, insect bites, and rarely bullous pemphigoid can also have limited clinical findings and severe pruritus.

Among the invisible dermatosis, one deserves special mention-scabies. Scabies is a common problem in elderly people because they are often in long-term care facilities or visit friends or family in such facilities. ${ }^{17}$ These congregative facilities often have ongoing or "imported" scabies outbreaks. In such outbreaks, $35 \%-50 \%$ of residents become infected. The facilities may not report the outbreak to the patient, so the exposure history is not available. Elderly patients can have exuberant or even hyperkeratotic infections or, at the other extreme, very subtle evidence of infection. The most useful clues in the history that should lead to a suspicion of scabies are the following: (1) the aforementioned potential exposure, (2) sudden onset of pruritus (patient can identify the week it started), (3) severity of pruritus is high (ie, 6-15 out of 10worst itch ever), and (4) pruritus spares the scalp or is minimal there despite severe itch elsewhere.

All pruritus tends to be worse at night, so nocturnal worsening is of minimal discriminatory value. In elderly people, the scabies mite no longer respects the "preferential areas" of involvement. The fingerwebs may be completely spared. The face may have lesions. In the patient with "invisible" scabies, the number of lesions may be very limited, and a careful search of the fingerwebs, genitalia, navel, breasts of women, axillary lines, and soles of the feet is paramount. In my experience the soles are a particularly "rich" area to look for burrows in elderly people. The elderly patient may not be able to see his or her soles and, if there is accompanying neuropathy, the patient may not sense pruritus from this region. Failure to remove an elderly patient's shoes may leave undiscovered the clue to the diagnosis. Because of impaired mobility, topical treatment of scabies in elderly people has a high failure rate. The patient simply cannot reach "every square inch" of their body surface. Unless the physician can guarantee adequate topical treatment, oral ivermectin should be considered in treating such patients. In the situation of hyperkeratotic scabies and florid disease, multiple weeks of treatment may be required, often combining topical and oral therapy. ${ }^{18}$ 


\section{Pruritus with No Rash}

In the patient with minimal skin lesions or only excoriations, the initial evaluation involves searching for metabolic causes of itch. Most patients with renal or hepatic itch have documented disease in those organ systems, and therefore reviewing the laboratories will usually result in identifying problems in these organ systems. (See "The Itch of Liver Disease" by Bergasa and "Pruritus and Renal Failure" by Berger and Steinhoff in this issue.) If these are normal hepatitis $C$ serology, serum calcium, serum iron and ferritin, a complete blood count $(\mathrm{CBC})$ with sedimentation rate, and thyroid function tests should be obtained. Iron deficiency should prompt evaluation for the cause of blood loss. If these tests are normal, evaluation should focus on detecting underlying malignancy. A physical examination should be performed, with the physician searching for lymphadenopathy. (See "Cancer and Itch" by Chiang et al in this issue.) In most cases, cancer-associated pruritus is caused by a lymphoreticular malignancy, especially Hodgkin's disease. Rarely, a solid tumor may be found. Eosinophilia on the CBC can be due to underlying malignancy, parasitic infestation, or hypereosinophilic syndrome (if $>1500$ absolute eosinophil count for $>6$ months). At times lymphocyte profiles will demonstrate abnormalities in the T-cell or B-cell number. If the cause of the eosinophilia is not determined, if there are abnormalities on the $\mathrm{CBC}$ that cannot be explained, if $\mathrm{T}$ - and $\mathrm{B}$-cell profiles are abnormal, and in particularly refractory patients, evaluation by an oncologist should be considered at this point.

\section{Pruritus with Rash}

Most patients older than the age of 60 who complain of pruritus have a visible dermatosis. At the University of California at San Francisco we reviewed the clinical morphology, biopsies, and eventual diagnostic outcome in a series of more than 100 elderly patients with a pruritic eruption. This is a selected population often referred by other dermatologists when standard approaches to treatment had failed. Scabies, bullous pemphigoid, and simple xerotic or stasis eczema were not common because these diagnoses had been made and effectively managed by the referring dermatologists. In the referred group, most of the patients had lesions that appeared morphologically as three patterns: eczematous plaques; erythematous papules; or lichenified papules (prurigo nodules) and plaques (lichen simplex chronicus). Often the patient had multiple morphologies. After a complete evaluation, five diagnoses encompassed $75 \%$ of the patients evaluated. The patients either had an eczematous dermatosis, lichen simplex/prurigo nodularis, subacute prurigo, transient acantholytic dermatosis, or a neuropathic disorder.

\section{Eczematous Dermatitis in the Elderly Patient}

Eczematous dermatitis was by far the most common morphology of the pruritic elderly patients in our series. A typical feature is accentuation of the dermatitis and pruritus in the patient's seborrheic keratoses. Removal of inflamed pruritic seborrheic keratoses, if limited in number, by curette can lead to reduction in the patient's symptoms. No specific cause for the eczematous eruption could be determined in approximately $25 \%$ of the patients. Contact dermatitis, num- mular dermatitis, and photodermatitis each represented approximately $15 \%$ of the eczematous cases. Atopy, stasis, and xerosis were approximately $10 \%$ each. The high rate of eczematous dermatitis in elderly people probably results from aging-associated epidermal barrier defects. Epidermal barrier defects also may increase the risk for the development of contact dermatitis, as suggested by the recent finding that nickel allergy is more common in persons who have filaggrin null mutations. Immunosenescense-associated preserved Th17 activity in elderly people may allow them to continue to develop allergic contact dermatitis. In addition, over many years elderly people are simply exposed to allergens more, increasing their risk for developing sensitization. Contact dermatitis is most frequent in elderly people on the lower extremities. The most common allergens reported are fragrances, topical medications, and lanolin. Topical corticosteroid allergy should also be considered. ${ }^{19}$

Although eczematous dermatitis is not a common reaction pattern for drug eruptions, calcium channel blockers (CCBs) may cause an eczematous dermatitis. In a study from France elderly patients with eczema were compared with patients of the same age without eczema, only the use of CCB was more common in the eczema group. Some patients had been on the medication for more than 10 years before the appearance of the eczema. In some cases of CCB-induced eczema, it took up to one year off the medication for the eczema to clear. ${ }^{20}$

\section{Chronic Itch-Scratch Disease in the Elderly Patient}

With persistent itching and scratching, lichenification and prurigo nodules result. In these patients the evaluation should include identifying the underlying cause of the pruritus, which is usually either a chronic eczematous dermatitis or a neurodegenerative disorder (brachioradial pruritus, for example).

\section{Papular Dermatitis in the Elderly Patient}

In $17 \%$ of pruritic elderly patients in our series the primary lesion was an urticarial edematous papule, often with a central excoriation. On biopsy these lesions show the features of a "dermal or urticarial hypersensitivity reaction" with scantto-moderate amounts of perivascular lymphocytes and at times interstitial eosinophils. Subacute prurigo is one term for these lesions. Some dermatologists simply refer to this as "red itchy bump disease." At times the biopsy will show eosinophilic infiltration primarily centered on the hair follicle in a pattern resembling "eosinophilic folliculitis." A subset of patients with this papular eruption (3\% in our series) had an underlying lymphoreticular malignancy. These cases would be correctly diagnosed as "eosinophilic disorder of myeloproliferative disease." ${ }^{21}$ The patients usually have chronic lymphocytic leukemia or myelodysplastic syndrome, but two patients in our series had smoldering leukemia with a normal CBC. (See "Cancer and itch" by Chiang et al in this issue.)

Approximately $15 \%$ of patients, $80 \%$ of them men, had transient acantholytic dermatitis (TAD). In some patients the TAD appeared first on the chest and back, followed by the urticarial papules on other sites. It is our hypothesis that these primarily papular eruptions are a consequence of "immunosenescence," which results in a Th2 dominant state in 
the skin. These elderly patients with primarily papular dermatoses resemble many HIV patients with pruritus.

It is not uncommon for the patient to have both papular and eczematous dermatitis simultaneously. Dr Grover originally recognized that in addition to TAD, patients frequently had xerotic eczema, contact dermatitis, and atopic dermatitis. ${ }^{22}$ The patient may either have separate eczematous plaques and urticarial papules or urticarial papules and plaques with minimal epidermal change, but some epidermal spongiosis on biopsy. Some of these patients eventuate in bullous pemphigoid. I agree with Dr Rietschel that those patients with primarily urticarial papules and plaques rarely have allergic contact dermatitis. ${ }^{23}$ The term urticarial dermatitis used in Australia may include patients with similar dermatoses, as this condition also preferentially affects the elderly. ${ }^{24}$

\section{Neurodegenerative Diseases}

Approximately $8 \%$ of our elderly pruritic patients proved to have a primarily neuropathic process. They usually presented with secondary lesions of lichenification (macular amyloid, lichen simplex, or prurigo nodularis). They often complained of both itching and other forms of dysesthesiaburning, tingling, or numbness. The patients frequently reported that all topical antiinflammatories were ineffective, but that application of ice brought relief. The most common clinical diagnoses were nostalgia paresthetica or brachioradial pruritus. ${ }^{25,26}$ Rubbing and scratching of the affected areas at times resulted in eczematous plaques. Some cases of genital pruritus are related to impingements and fall into this category. ${ }^{27}$

At times pruritus was more diffuse and related to a generalized neuropathy. Diabetic neuropathy is associated with generalized truncal pruritus and may explain the association between diabetes and pruritus. ${ }^{14}$ In the patient with generalized pruritus and secondary skin lesions of scratching only, a primarily neuropathic process of the peripheral or central nervous system should be sought. Neurologists and physical medicine physicians can be useful in the evaluation of such patients. (See "Neuropathic Itch" by Oaklander in this issue.)

\section{Unifying Hypothesis- Eruptions of Senescence}

A useful way to think about the pruritic elderly patient is to follow the evaluation scheme outlined previously. A careful evaluation for medication exposure, especially CCBs, is important. Underlying neurological disease should be sought. When the patient is found to have primarily skin conditions in the most common categories (eczematous dermatitis, itchscratch dermatosis, and papular pruritic eruptions), we may use the diagnosis of "eruptions of senescence" in the medical record. If the primary morphology is eczematous, the primary problem is a barrier defect. If the primary morphology is papular, then there is primarily an immunologic problem-immunosenescense. However, in most of these pa- tients, multiple types of lesions coexist or are found over time. In men, Grover's disease is also frequently present in those patients with eczematous dermatitis or other papular eruptions. By considering these disorders all a consequence of the physiological changes of age, we can explain why the patient has the eruptions and what factors are responsible for each of the specific morphologic patterns. It also explains why patients may have multiple different morphologies at the same time or over sequential visits.

\section{Treatment}

For the patient with a primarily eczematous process, medications, contactants, and photosensitivity must be considered as causal or coexistent factors contributing to the dermatitis. Patch testing and phototesting may be required for diagnosis. For the most part the eczematous patients have barrier failure as a major contributing factor. For treatment the "soak and smear" approach can be very effective. Soaking in a warm tub for 15 minutes and applying a class I or class II steroid diluted 1:4 in a heavy moisturizer (i. e., Vanicream, Petrolatum, Aquaphor, Eucerin) onto the wet skin followed by an occlusion suit or wet wraps can be very effective. Antihistamines for sedation can be useful at this stage. If this fails, a short course of steroids may be required to calm down the dermatitis. Phototherapy or even day treatment with tar and ultraviolet phototherapy can be considered in refractory cases. If the dermatitis is chronic and refractory to brief courses of systemic steroids and phototherapy, low-dose immunosuppressives may be useful. Azathioprine, mycophenolate, or methotrexate can all be effective, the agent used based upon the patients risk profile and medication availability. Sometimes, after the barrier has been repaired with aggressive topical treatment, and the immune reaction has been calmed, the systemic immunosuppressive can be removed and the patient maintained with topical treatment or phototherapy.

In the patients with the primarily papular morphologythe red itchy bump patient-soak and smear can be used as an initial treatment. However, in this group, phototherapy is usually quite effective, both for induction and maintenance of remission. Because the immunosenescence that drives the papular lesions is not reversible, often chronic treatment with either phototherapy or an immunomodulator is required.

Grover's disease often can be treated with topical steroids. If the TAD is not pruritic, the patient can be reassured that treatment is not required. In severe cases, oral retinoids can be attempted, but if there is an associated eczematous dermatitis, that could be exacerbated.

In patients with refractory prurigo nodularis, phototherapy, day treatment, and neurally acting antipruritic agents can be effective. Gabapentin, duloxetine, mirtazepine alone, or gabapentin plus mirtazepine can be considered in refractory patients. Thalidomide is especially useful in the patient with severe prurigo nodularis with no associated background dermatitis.

In addition to specific treatment for the skin, oral antipruritic agents can be used. The agent chosen would be deter- 
mined by the nature of the dermatitis. Agents that have significant effect on the nervous system-tricyclics, gabapentin, duloxetine, and thalidomide-are useful in neuropathic pruritus. Standard sedating first-generation antihistamines can be used, but with caution in elderly people. Excess sedation, increased risk of falling, confusion, and urinary retention are all complications for which elderly people are particularly at risk. (See "Pruritus: Management Algorithms and Experimental Therapies" by Steinhoff et al in this issue.)

In summary, elderly people are significantly affected by pruritic dermatoses. This burden of disease in elderly people is a consequence of defects or disorders of the three major players that produce or mediate pruritus - the epidermis, the nervous system, and the immune system. Senescent barrier failure, immunosenescence, and neurodegenerative disorders all afflict elderly people and can produce pruritus. The pruritic conditions produced by these age-related processes can be considered the "eruptions of senescence." Treatment directed at the primary element triggering the pruritus is most effective. At times the pruritic elderly patient will have skin lesions induced by more than one of these age related processes, and treatment for each pathogenic process may be required.

\section{References}

1. Reich A, Ständer S, Szepietowski JC: Pruritus in the elderly. Clin Dermatol 29:15-23, 2011

2. Candore G, Caruso C, Jirillo E, et al: Low grade inflammation as a common pathogenetic denominator in age-related diseases: Novel drug targets for anti-ageing strategies and successful ageing achievement. Curr Pharm Des 16:584-596, 2010

3. Pawelec G, Larbi A, Derhovanessian E: Senescence of the human immune system. J Comp Pathol 142 Suppl 1:S39-S44, 2010

4. Weiskopf D, Weinberger B, Grubeck-Loebenstein B: The aging of the immune system. Transpl Int 22:1041-1050, 2009

5. Ferrando-Martínez S, Franco JM, Hernandez A, et al: Thymopoiesis in elderly human is associated with systemic inflammatory status. Age (Dordr) 31:87-97, 2009

6. Sandmand M, Bruunsgaard H, Kemp K, et al: Is ageing associated with a shift in the balance between type 1 and type 2 cytokines in humans? Clin Exp Immunol 127:107-114, 2002

7. Haynes L, Maue AC: Effects of aging on T cell function. Curr Opin Immunol 21:414-417, 2009

8. Choi EH, Man MQ, Xu P, et al: Stratum corneum acidification is im- paired in moderately aged human and murine skin. J Invest Dermatol 127:2847-2856, 2007

9. Jensen JM, Förl M, Winoto-Morbach S, et al: Acid and neutral sphingomyelinase, ceramide synthase, and acid ceramidase activities in cutaneous aging. Exp Dermatol 14:609-618, 2005

10. Li J, Tang H, Hu X, et al: Aquaporin-3 gene and protein expression in sun-protected human skin decreases with skin ageing. Australas J Dermatol 51:106-112, 2010

11. White-Chu EF, Reddy M: Dry skin in the elderly: Complexities of a common problem. Clin Dermatol 29:37-42, 2011

12. Veien NK, Laurberg G: Brachioradial pruritus: A follow-up of 76 patients. Acta Derm Venereol 91:183-185, 2011

13. Canavero S, Bonicalzi V, Massa-Micon B: Central neurogenic pruritus: A literature review. Acta Neurol Belg 97:244-247, 1997

14. Yamaoka H, Sasaki H, Yamasaki H, et al: Truncal pruritus of unknown origin may be a symptom of diabetic polyneuropathy. Diabetes Care 33:150-155, 2010

15. Kamann S, Flaig MJ, Korting HC: Hydroxyethyl starch-induced itch: Relevance of light microscopic analysis of semi-thin sections and electron microscopy. J Dtsch Dermatol Ges 5:204-208, 2007

16. Bork K: Pruritus precipitated by hydroxyethyl starch: A review. Br J Dermatol 152:3-12, 2005

17. Bouvresse S, Chosidow O: Scabies in healthcare settings. Curr Opin Infect Dis 23:111-118, 2010

18. Currie BJ, McCarthy JS: Permethrin and ivermectin for scabies. N Engl J Med 362:717-725, 2010

19. Prakash AV, Davis MD: Contact dermatitis in older adults: A review of the literature. Am J Clin Dermatol 11:373-381, 2010

20. Joly P, Benoit-Corven C, Baricault S, et al: Chronic eczematous eruptions of the elderly are associated with chronic exposure to calcium channel blockers: Results from a case-control study. J Invest Dermatol 127:2766-2771, 2007

21. Byrd JA, Scherschun L, Chaffins ML, et al: Eosinophilic dermatosis of myeloproliferative disease: Characterization of a unique eruption in patients with hematologic disorders. Arch Dermatol 137:1378-1380, 2001

22. Grover RW, Rosenbaum R: The association of transient acantholytic dermatosis with other skin diseases. J Am Acad Dermatol 11:253-256, 1984

23. Rietschel RL: A clinician's view of urticarial dermatitis. Arch Dermatol 142:932, 2006

24. Kossard S, Hamann I, Wilkinson B: Defining urticarial dermatitis: A subset of dermal hypersensitivity reaction pattern. Arch Dermatol 142: 29-34, 2006

25. Yilmaz S, Ceyhan AM, Baysal Akkaya V: Brachioradial pruritus successfully treated with gabapentin. J Dermatol 37:662-665, 2010

26. De Ridder D, Hans G, Pals P, et al: A C-fiber-mediated neuropathic brachioradial pruritus. J Neurosurg 113:118-121, 2010

27. Koh WL, Liu TT: A cured patient who came back for consultation: Neuropathic scrotal pruritus relieved after ipsilateral inguinal hernia repair. Int J STD AIDS 21:658-659, 2010 